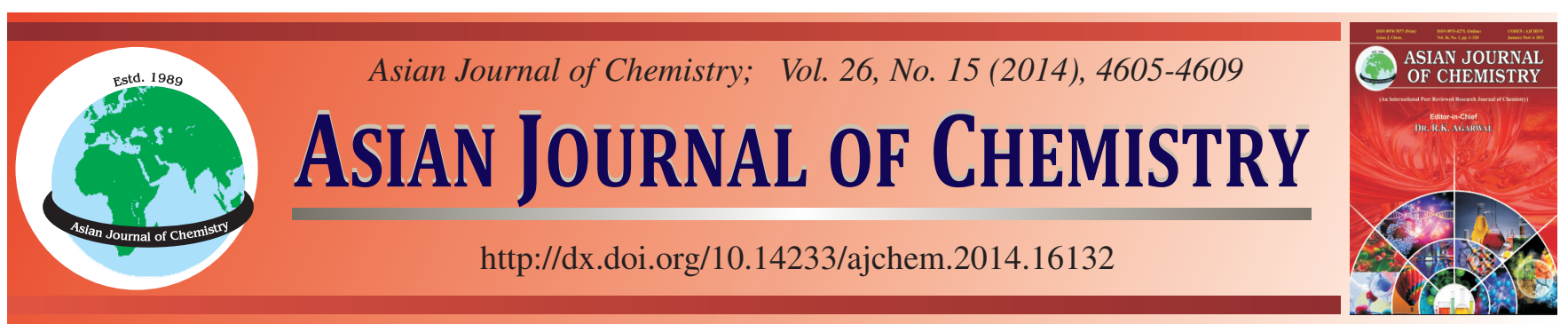

\title{
Synthesis, Characterization and Urease Inhibiting Derivatives of 5-(3,4-Methylenedioxyphenyl)-1,3,4-Oxadiazol-2-thiol
}

\author{
Aziz-Ur-Rehman ${ }^{1, *}$, Asia Siddiqa ${ }^{1}$, M. Athar Abbasi ${ }^{1}$, Shahid Rasool ${ }^{1}$, \\ M. NADEem Akhtar ${ }^{2}$, M. Arif LODhi ${ }^{3}$, Khadija NAFeESA ${ }^{1}$ and AJmal Khan ${ }^{4}$
}

\begin{abstract}
${ }^{1}$ Department of Chemistry, Government College University, Lahore-54000, Pakistan
${ }^{2}$ Faculty of Industrial Sciences \&and Technology, University Malaysia Pahang, Lebuhraya Tun Razak 26300, Gambang, Kuantan, Malaysia

${ }^{3}$ Department of Biochemistry, Abdul Wali Khan University, Mardan, KPK-23200, Pakistan

${ }^{4}$ Department of Chemistry, University of Malakand, Kyber Pakhtunkhwa, Pakistan
\end{abstract}

*Corresponding author: Tel: +92 42 111000010; Ext. 450; E-mail: azizryk@yahoo.com; rehman@gcu.edu.pk

\begin{abstract}
In the present work, the urease inhibition activity of 1,3,4-oxadiazole bearing molecules was evaluated and were found to be potential inhibitors. 3,4-(Methylenedioxy)benzoic acid (1) was employed to synthesize 5-(3,4-methylenedioxyphenyl)-1,3,4-oxadiazol-2-thiol (4) via a series of steps. It was further stepped to yield S-substituted-5-(3,4-methylenedioxyphenyl)-1,3,4-oxadiazole derivatives (6a-h) on reaction with alkyl/aralkyl halides (5a-h) in DMF using LiH as an activator. All the synthesized compounds were well supported by IR, ${ }^{1} \mathrm{H}$ NMR and EIMS spectral analysis. The enzyme inhibition activity against urease enzyme showed these molecules as potent inhibitors of this enzyme.
\end{abstract}

Keywords: 3,4-(Methylenedioxy)benzoic acid, 1,3,4-Oxadiazole, Urease, ${ }^{1} \mathrm{H}$ NMR and EIMS.

\section{INTRODUCTION}

Because of bacterial drug resistance, the scientists are attempting to inaugurate new potent compounds which may be employed for the cure of various diseases ${ }^{1}$. Most of the researches are working on heterocyclic compounds in this regard $^{2}$. Oxadiazole nucleus has attributed much attention because to their pharmacological importance especially 1,3,4oxadiazoles $^{3-6}$. Benzodioxole moiety bears a crucial importance among the potential drugs. Many anticancer, antidepressant etc. drugs possess this moiety in their structures ${ }^{7-8}$.

Urease (EC 3.5.1.5) is urea amidohydrolase and has an important role in pathogenic processes taking place in humans and animals. It has the main part in the cause of urolithiasis, pyelonephritis, urinary catheter incrustation, peptic ulceration, kidney stone and hepatic encephalopathy ${ }^{9-11}$.

The need of the hour is to introduce potent molecules that may be used as remedy for various complaints. Relating to our research projects ${ }^{12-13}$, the S-substitution of 5-(3,4methylenedioxyphenyl)-1,3,4-oxadiazol-2-thiol bearing benzodioxole moiety was carried out with an aim of preparing new potent urease inhibitors with low toxicity.

\section{EXPERIMENTAL}

Chemicals were labeled by Alfa Aesar and Sigma-Aldrich with extra purification and analytical grade solvents were purchased from local suppliers. Melting points were computed on a Griffin and George apparatus employing open capillary tube and were uncorrected. Purity of the synthesized molecules was assessed by thin layer chromatography (TLC) using precoated silica gel G-25-UV 254 plates with solvent system (n$\mathrm{C}_{6} \mathrm{H}_{14}: \mathrm{CH}_{3} \mathrm{COOC}_{2} \mathrm{H}_{5}$ ) with single spot under UV at $254 \mathrm{~nm}$. The I.R. spectra were figured on a Jasco-320-A spectrophotometer with $\mathrm{KBr}$ pellet and the unit used for $(\bar{v})$ is $\mathrm{cm}^{-1} .{ }^{1} \mathrm{H}$ NMR spectra were figured on a Bruker spectrometers operating at $300 / 400 \mathrm{MHz}$ in $\mathrm{CDCl}_{3}$. Chemical shifts are given in ppm with TMS as reference standard. Mass spectra (EIMS) were figured on a JMS-HX-110 spectrometer, with a data system.

Synthesis of ethyl 3,4-(methylene-dioxy)benzoate (2): 3,4-(Methylenedioxy)benzoic acid (1, $5 \mathrm{~g}$ ) was homogenized in $99 \%$ ethanol $(20 \mathrm{~mL})$ in a $250 \mathrm{~mL}$ round bottom flask. $2.5 \mathrm{~mL}$ conc. $\mathrm{H}_{2} \mathrm{SO}_{4}$ was added and the mixture was refluxed for $3 \mathrm{~h}$. TLC was developed for confirmation of reaction completion. After completion, the ester was extracted by solvent 
extraction using $50 \mathrm{~mL} \mathrm{CHCl}_{3}$ from a separating funnel after the addition of $150 \mathrm{~mL}$ distilled water. Before the separation of organic layer, the aq. $\mathrm{Na}_{2} \mathrm{CO}_{3}$ solution was poured to neutralize the mixture up to $\mathrm{pH}$ of 9-10. This step converted untreated organic and left sulphuric acid into salts washed away by aqueous layer. Chloroform was distilled off to collect yellowish transparent ester 2. Yellowish transparent liquid; Yield: $90 \%$; m.f.: $\mathrm{C}_{10} \mathrm{H}_{10} \mathrm{O}_{4}$; Molecular mass: 194; IR (KBr, $\left.v_{\max }, \mathrm{cm}^{-1}\right): 3006$ (aromatic C-H stretching), $1710(\mathrm{C}=\mathrm{O}$ ester stretching), 1605 (Ar C=C stretching), 1110 (C-O ester stretching); ${ }^{1} \mathrm{H} \mathrm{NMR}\left(\mathrm{CDCl}_{3}, 400 \mathrm{MHz}, \delta / \mathrm{ppm}\right): 7.64$ (dd, $J=$ 8.4, 1.6 Hz, 1H, H-6'), 7.44 (d, J = 1.2 Hz, 1H, H-2'), 6.82 (d, $\left.J=8.0 \mathrm{~Hz}, 1 \mathrm{H}, \mathrm{H}-5^{\prime}\right), 6.01$ (s, 2H, H-7'), 4.32 (q, $J=7.2 \mathrm{~Hz}$, $\left.2 \mathrm{H},-\mathrm{OCH}_{2} \mathrm{CH}_{3}\right), 1.37$ (t, $\left.J=7.2 \mathrm{~Hz}, 3 \mathrm{H},-\mathrm{OCH}_{2} \mathrm{CH}_{3}\right)$; EIMS $(\mathrm{m} / \mathrm{z}): 194[\mathrm{M}]^{\circ+}, 165\left[\mathrm{C}_{8} \mathrm{H}_{5} \mathrm{O}_{4}\right]^{+}, 149\left[\mathrm{C}_{8} \mathrm{H}_{5} \mathrm{O}_{3}\right]^{+}, 121\left[\mathrm{C}_{7} \mathrm{H}_{5} \mathrm{O}_{2}\right]^{+}$, $29\left[\mathrm{C}_{2} \mathrm{H}_{5}\right]^{+}$.

Synthesis of 3,4-(methylenedioxy)-benzohydrazide (3): The molecule $2(0.1 \mathrm{~mol})$ was diluted in $250 \mathrm{~mL}$ round bottom flask with $100 \mathrm{~mL}$ ethanol and followed by addition of $10 \mathrm{~mL}$ $80 \% \mathrm{~N}_{2} \mathrm{H}_{4} \cdot \mathrm{H}_{2} \mathrm{O}$. Refluxing was continued for $6 \mathrm{~h}$. After monitoring by TLC, ice cold distilled $\mathrm{H}_{2} \mathrm{O}$ was poured to acquire the precipitates (3) which were filtered and washed off with distilled $\mathrm{H}_{2} \mathrm{O}$. White amorphous solid; Yield: $97 \%$; m.p: $170{ }^{\circ} \mathrm{C}$; m.f.: $\mathrm{C}_{8} \mathrm{H}_{8} \mathrm{~N}_{2} \mathrm{O}_{3}$; Molecular mass: 180; IR ( $\left.\mathrm{KBr}, \mathrm{v}_{\max }, \mathrm{cm}^{-1}\right)$ : 3250 (NH stretching), 3010 (aromatic C-H stretching), 1730 ( $\mathrm{C}=\mathrm{O}$ amide stretching), 1610 (Ar $\mathrm{C}=\mathrm{C}$ stretching); ${ }^{1} \mathrm{H} \mathrm{NMR}$ $\left(\mathrm{CDCl}_{3}, 400 \mathrm{MHz}, \delta / \mathrm{ppm}\right): 7.66$ (dd, $J=8.4,1.6 \mathrm{~Hz}, 1 \mathrm{H}$, H-6') 7.45 (d, $\left.J=1.2 \mathrm{~Hz}, 1 \mathrm{H}, \mathrm{H}-2^{\prime}\right), 6.87$ (d, $J=8.0 \mathrm{~Hz}, 1 \mathrm{H}$, H-5'), 6.06 (s, 2H, H-7'); EIMS $(\mathrm{m} / z)$ : $180[\mathrm{M}]^{\circ+}, 164$ $\left[\mathrm{C}_{8} \mathrm{H}_{6} \mathrm{NO}_{3}\right]^{+}, 149\left[\mathrm{C}_{8} \mathrm{H}_{5} \mathrm{O}_{3}\right]^{+}, 121\left[\mathrm{C}_{7} \mathrm{H}_{5} \mathrm{O}_{2}\right]^{+}$.

Synthesis of 5-(3,4-methylene-dioxyphenyl)-1,3,4oxadiazol-2-thiol (4): The product $3(0.1 \mathrm{~mol})$ was shaken well with $100 \mathrm{~mL}$ absolute ethanol in $250 \mathrm{~mL}$ round bottom flask and set to reflux after the addition of $\mathrm{CS}_{2}(0.3 \mathrm{~mol})$ and $\mathrm{KOH}(0.2 \mathrm{~mol})$ for $5 \mathrm{~h}$. After the final TLC, $300 \mathrm{~mL}$ distilled water was added along with dil. $\mathrm{HCl}$ to make $\mathrm{pH}$ of 3-4 to acquire the precipitates of 4 . The addition of acid is crucial to change back the salt form of 5-(3,4-methylene-dioxyphenyl)1,3,4-oxadiazol-2-thiol into acidic one but limited amount because excess reduces the amount of product. The precipitates were filtered, washed with dist. $\mathrm{H}_{2} \mathrm{O}$ and re-crystallized from $\mathrm{CH}_{3} \mathrm{OH}$. White amorphous solid; Yield: $83 \%$; m.p: $238{ }^{\circ} \mathrm{C}$; m.f.: $\mathrm{C}_{9} \mathrm{H}_{6} \mathrm{~N}_{2} \mathrm{O}_{3} \mathrm{~S}$; Molecular mass: 222; IR ( $\left.\mathrm{KBr}, \mathrm{v}_{\max }, \mathrm{cm}^{-1}\right)$ : 3011 (aromatic $\mathrm{C}-\mathrm{H}$ stretching), 1613 ( $\mathrm{Ar} \mathrm{C}=\mathrm{C}$ stretching), 1575 (C=N stretching), 1109 (C-O-C stretching); ${ }^{1} \mathrm{H}$ NMR $\left(\mathrm{CDCl}_{3}, 400 \mathrm{MHz}, \delta / \mathrm{ppm}\right): 7.52(\mathrm{dd}, J=8.4,1.2 \mathrm{~Hz}, 1 \mathrm{H}$, H-6'), 7.44 (s, 1H, H-2'), 6.89 (d, J = 8.4 Hz, 1H, H-5'), 6.06 (s, 2H, H-7'); $\operatorname{EIMS}(\mathrm{m} / \mathrm{z}): 236[\mathrm{M}]^{\bullet+}, 221\left[\mathrm{C}_{9} \mathrm{H}_{5} \mathrm{~N}_{2} \mathrm{O}_{3} \mathrm{~S}\right]^{+}$, $189\left[\mathrm{C}_{9} \mathrm{H}_{5} \mathrm{~N}_{2} \mathrm{O}_{3}\right]^{+}, 163\left[\mathrm{C}_{8} \mathrm{H}_{5} \mathrm{NO}_{3}\right]^{\bullet+}, 147\left[\mathrm{C}_{8} \mathrm{H}_{5} \mathrm{NO}_{2}\right]^{\bullet+}, 121$ $\left[\mathrm{C}_{7} \mathrm{H}_{5} \mathrm{O}_{2}\right]^{+}$.

General procedure for the synthesis of S-substituted derivatives (6a-h): The compound 4 (0.2 g, 0.009 mol) was homogenized in $15 \mathrm{~mL}$ DMF in a $100 \mathrm{~mL}$ round bottom flask and then $\mathrm{LiH}(0.004 \mathrm{~g})$ was added along with stirring. The electrophiles i.e. alkyl/aralkyl halides (5a-h, 0.009 mol) were poured into homogeneous solution after $0.25 \mathrm{~h}$ and further stirred for 4-6 h. After single spot by TLC, ice cold dist. water was introduced into reaction contents along with aq. $\mathrm{Na}_{2} \mathrm{CO}_{3}$ up to $\mathrm{pH}$ of 9-10 and the precipitates (6a-h) were acquired.
The mixture is basified to get rid of the untreated 4. Precipitates were filtered, washed with distilled $\mathrm{H}_{2} \mathrm{O}$ and dried.

2-(2-Bromoethyl)thio-5-(3,4-methylenedioxyphenyl)1,3,4-oxadiazole (6a): Creamy white amorphous solid; Yield: 90 \%; m.p: $122{ }^{\circ} \mathrm{C}$; m.f.: $\mathrm{C}_{11} \mathrm{H}_{9} \mathrm{~N}_{2} \mathrm{O}_{3} \mathrm{SBr}$; Molecular mass: 329 ; IR $\left(\mathrm{KBr}, \mathrm{v}_{\max }, \mathrm{cm}^{-1}\right): 3014$ (aromatic C-H stretching), 1616 (Ar C=C stretching), 1579 (C=N stretching), 1107 (C-O-C stretching); ${ }^{1} \mathrm{H} \mathrm{NMR}\left(\mathrm{CDCl}_{3}, 400 \mathrm{MHz}, \delta / \mathrm{ppm}\right): 7.53$ (dd, $J=$ 8.0, 1.2 Hz, 1H, H-6'), 7.43 (d, J = 1.2 Hz, 1H, H-2'), 6.89 (d, $\left.J=8.0 \mathrm{~Hz}, 1 \mathrm{H}, \mathrm{H}-5^{\prime}\right), 6.04$ (s, 2H, H-7'), 3.76 (t, $J=7.2 \mathrm{~Hz}$, 2H, H-2"), 3.68 (t, $J=7.2 \mathrm{~Hz}, 2 \mathrm{H}, \mathrm{H}-1 ") ; \operatorname{EIMS}(\mathrm{m} / \mathrm{z}): 331[\mathrm{M}$ $+2]^{\bullet+}, 329[\mathrm{M}]^{\bullet+}, 221\left[\mathrm{C}_{9} \mathrm{H}_{5} \mathrm{~N}_{2} \mathrm{O}_{3} \mathrm{~S}\right]^{+}, 189\left[\mathrm{C}_{9} \mathrm{H}_{5} \mathrm{~N}_{2} \mathrm{O}_{3}\right]^{+}, 163$ $\left[\mathrm{C}_{8} \mathrm{H}_{5} \mathrm{NO}_{3}\right]^{\bullet+}, 147\left[\mathrm{C}_{8} \mathrm{H}_{5} \mathrm{NO}_{2}\right]^{\bullet+}, 121\left[\mathrm{C}_{7} \mathrm{H}_{5} \mathrm{O}_{2}\right]^{+}, 108\left[\mathrm{C}_{2} \mathrm{H}_{4} \mathrm{Br}\right]^{+}$, $28\left[\mathrm{C}_{2} \mathrm{H}_{4}\right]^{+}$.

2-(2-Chloroethyl)thio-5-(3,4-methylenedioxyphenyl)1,3,4-oxadiazole (6b): White amorphous solid; Yield: $90 \%$; m.p: $130{ }^{\circ} \mathrm{C}$; m.f.: $\mathrm{C}_{11} \mathrm{H}_{9} \mathrm{~N}_{2} \mathrm{O}_{3} \mathrm{SCl}$; Molecular mass: 284; IR $\left(\mathrm{KBr}, \mathrm{v}_{\max }, \mathrm{cm}^{-1}\right): 3285$ (aromatic C-H stretching), 1630 (Ar $\mathrm{C}=\mathrm{C}$ stretching $), 1576(\mathrm{C}=\mathrm{N}$ stretching $), 1109$ (C-O-C stretching); ${ }^{1} \mathrm{H} \mathrm{NMR}\left(\mathrm{CDCl}_{3}, 400 \mathrm{MHz}, \delta / \mathrm{ppm}\right): 7.53$ (dd, $J=$ 8.0, 1.6 Hz, 1H, H-6'), 7.43 (d, J = 1.6 Hz, 1H, H-2'), 6.87 (d, $\left.J=8.0 \mathrm{~Hz}, 1 \mathrm{H}, \mathrm{H}-5^{\prime}\right), 6.04$ (d, $\left.J=2.4 \mathrm{~Hz}, 2 \mathrm{H}, \mathrm{H}-7^{\prime}\right), 3.93$ (t, $J$ $\left.=7.2 \mathrm{~Hz}, 2 \mathrm{H}, \mathrm{H}-2^{\prime \prime}\right), 3.61$ (t, $\left.J=7.2 \mathrm{~Hz}, 2 \mathrm{H}, \mathrm{H}-1 "\right)$; EIMS $(\mathrm{m} / \mathrm{z}): 286[\mathrm{M}+2]^{\bullet+}, 284[\mathrm{M}]^{\bullet+}, 221\left[\mathrm{C}_{9} \mathrm{H}_{5} \mathrm{~N}_{2} \mathrm{O}_{3} \mathrm{~S}\right]^{+}, 189$ $\left[\mathrm{C}_{9} \mathrm{H}_{5} \mathrm{~N}_{2} \mathrm{O}_{3}\right]^{+}, 163\left[\mathrm{C}_{8} \mathrm{H}_{5} \mathrm{NO}_{3}\right]^{\bullet+}, 147\left[\mathrm{C}_{8} \mathrm{H}_{5} \mathrm{NO}_{2}\right]^{\bullet+}, 121$ $\left[\mathrm{C}_{7} \mathrm{H}_{5} \mathrm{O}_{2}\right]^{+}, 63\left[\mathrm{C}_{2} \mathrm{H}_{4} \mathrm{Cl}\right]^{+}, 28\left[\mathrm{C}_{2} \mathrm{H}_{4}\right]^{+}$.

2-(Ethoxycarbonyl)methylthio-5-(3,4-methylenedioxyphenyl)-1,3,4-oxadiazole (6c): White amorphous solid; Yield: $95 \%$; m.p: $116^{\circ}$ C; m.f.: $\mathrm{C}_{13} \mathrm{H}_{12} \mathrm{~N}_{2} \mathrm{O}_{5} \mathrm{~S}$; Molecular mass: 308; IR ( $\left.\mathrm{KBr}, v_{\max }, \mathrm{cm}^{-1}\right): 3020$ (aromatic C-H stretching), 1715 ( $\mathrm{C}=\mathrm{O}$ stretching), 1617 (Ar $\mathrm{C}=\mathrm{C}$ stretching), $1576(\mathrm{C}=\mathrm{N}$ stretching), 1106 (C-O-C stretching); ${ }^{1} \mathrm{H}$ NMR $\left(\mathrm{CDCl}_{3}, 400\right.$ $\mathrm{MHz}, \delta / \mathrm{ppm}$ ): 7.52 (dd, $J=8.4,1.6 \mathrm{~Hz}, 1 \mathrm{H}, \mathrm{H}-6$ '), 7.42 (d, $\left.J=1.2 \mathrm{~Hz}, 1 \mathrm{H}, \mathrm{H}-2^{\prime}\right), 6.89$ (d, $\left.J=8.0 \mathrm{~Hz}, 1 \mathrm{H}, \mathrm{H}-5^{\prime}\right), 6.03$ (s, $2 \mathrm{H}, \mathrm{H}-7$ '), 4.26 (q, J = 7.2 Hz, 2H, H-3"), 4.06 (s, 2H, H-1"), 1.29 (t, $J=7.2 \mathrm{~Hz}, 3 \mathrm{H}, \mathrm{H}-4 ")$; $\operatorname{EIMS}(\mathrm{m} / z): 308[\mathrm{M}]^{\circ+}, 221$ $\left[\mathrm{C}_{9} \mathrm{H}_{5} \mathrm{~N}_{2} \mathrm{O}_{3} \mathrm{~S}\right]^{+}, 189\left[\mathrm{C}_{9} \mathrm{H}_{5} \mathrm{~N}_{2} \mathrm{O}_{3}\right]^{+}, 163\left[\mathrm{C}_{8} \mathrm{H}_{5} \mathrm{NO}_{3}\right]^{\bullet+}, 147$ $\left[\mathrm{C}_{8} \mathrm{H}_{5} \mathrm{NO}_{2}\right]^{\bullet+}, 121\left[\mathrm{C}_{7} \mathrm{H}_{5} \mathrm{O}_{2}\right]^{+}, 87\left[\mathrm{C}_{4} \mathrm{H}_{7} \mathrm{O}_{2}\right]^{+}, 42\left[\mathrm{C}_{2} \mathrm{H}_{2} \mathrm{O}\right]^{+}$.

2-Benzylthio-5-(3,4-methylenedioxyphenyl)-1,3,4oxadiazole (6d): White amorphous solid; Yield: $86 \%$; m.p: $130{ }^{\circ} \mathrm{C}$; m.f.: $\mathrm{C}_{16} \mathrm{H}_{12} \mathrm{~N}_{2} \mathrm{O}_{3} \mathrm{~S}$; Molecular mass: 312; IR (KBr, $V_{\max }, \mathrm{cm}^{-1}$ ): 3285 (aromatic C-H stretching), 1630 (Ar C=C stretching), 1588 (C=N stretching), 1109 (C-O-C stretching); ${ }^{1} \mathrm{H} \mathrm{NMR}\left(\mathrm{CDCl}_{3}, 400 \mathrm{MHz}, \delta / \mathrm{ppm}\right): 7.51(\mathrm{dd}, J=7.2,1.2 \mathrm{~Hz}$, 1H, H-6'), 7.44 (brd.s, 1H, H-2'), 7.41 (d, J=7.2 Hz, 2H, H-2", H-6"), 7.34-7.30 (m, 3H, H-3" to H-5"), 6.88 (d, J=7.2 Hz, 1H, H-5'), 6.03 (s, 2H, H-7'), 4.48 (s, 2H, H-7"); EIMS (m/z): 312 $[\mathrm{M}]^{\bullet+}, 221\left[\mathrm{C}_{9} \mathrm{H}_{5} \mathrm{~N}_{2} \mathrm{O}_{3} \mathrm{~S}^{+}, 189\left[\mathrm{C}_{9} \mathrm{H}_{5} \mathrm{~N}_{2} \mathrm{O}_{3}\right]^{+}, 163\left[\mathrm{C}_{8} \mathrm{H}_{5} \mathrm{NO}_{3}\right]^{\bullet+}\right.$, $147\left[\mathrm{C}_{8} \mathrm{H}_{5} \mathrm{NO}_{2}\right]^{0+}, 121\left[\mathrm{C}_{7} \mathrm{H}_{5} \mathrm{O}_{2}\right]^{+}, 122\left[\mathrm{C}_{7} \mathrm{H}_{6} \mathrm{~S}\right]^{+}, 91\left[\mathrm{C}_{7} \mathrm{H}_{7}\right]^{\bullet+}, 77$ $\left[\mathrm{C}_{6} \mathrm{H}_{5}\right]^{*+}, 65\left[\mathrm{C}_{5} \mathrm{H}_{5}\right]^{+}, 51\left[\mathrm{C}_{4} \mathrm{H}_{3}\right]^{\bullet+}$.

2-(2-Methylbenzyl)thio-5-(3,4-methylenedioxyphenyl)1,3,4-oxadiazol (6e): White amorphous solid; Yield: $84 \%$; m.p: $106{ }^{\circ} \mathrm{C}$; m.f.: $\mathrm{C}_{17} \mathrm{H}_{14} \mathrm{~N}_{2} \mathrm{O}_{3} \mathrm{~S}$; Molecular mass: 326; IR $\left(\mathrm{KBr}, v_{\max }, \mathrm{cm}^{-1}\right.$ ): 3294 (aromatic C-H stretching), 1627 (Ar $\mathrm{C}=\mathrm{C}$ stretching $), 1591(\mathrm{C}=\mathrm{N}$ stretching $), 1108$ (C-O-C stretching); ${ }^{1} \mathrm{H} \mathrm{NMR}\left(\mathrm{CDCl}_{3}, 400 \mathrm{MHz}, \delta / \mathrm{ppm}\right): 7.52$ (dd, $J=$ 8.4, 1.6 Hz, 1H, H-6'), 7.43 (d, J = 1.6 Hz, 1H, H-2'), 7.38 (d, $J=7.2 \mathrm{~Hz}, 1 \mathrm{H}, \mathrm{H}-6 "), 7.19-7.12$ (m, 2H, H-4", H-5"), 6.89 (d, $J=8.0 \mathrm{~Hz}, 1 \mathrm{H}, \mathrm{H}-5$ '), 6.06 (d, $J=6.4 \mathrm{~Hz}, 1 \mathrm{H}, \mathrm{H}-3 "), 6.03$ (s, 
2H, H-7'), 4.52 (s, 2H, H-7"), 2.43 (s, 3H, $\left.\mathrm{CH}_{3}-2 "\right)$; $\operatorname{EIMS~(m/z):~}$ $326[\mathrm{M}]^{\circ+}, 221\left[\mathrm{C}_{9} \mathrm{H}_{5} \mathrm{~N}_{2} \mathrm{O}_{3} \mathrm{~S}\right]^{+}, 189\left[\mathrm{C}_{9} \mathrm{H}_{5} \mathrm{~N}_{2} \mathrm{O}_{3}\right]^{+}, 163\left[\mathrm{C}_{8} \mathrm{H}_{5} \mathrm{NO}_{3}\right]^{\circ+}$, $147\left[\mathrm{C}_{8} \mathrm{H}_{5} \mathrm{NO}_{2}\right]^{\bullet+}, 137\left[\mathrm{C}_{8} \mathrm{H}_{9} \mathrm{~S}\right]^{+}, 121\left[\mathrm{C}_{7} \mathrm{H}_{5} \mathrm{O}_{2}\right]^{+}, 106\left[\mathrm{C}_{8} \mathrm{H}_{9}\right]^{+}$, $90\left[\mathrm{C}_{7} \mathrm{H}_{6}\right]^{\bullet+}, 77\left[\mathrm{C}_{6} \mathrm{H}_{5}\right]^{+}, 65\left[\mathrm{C}_{5} \mathrm{H}_{5}\right]^{\bullet+}, 51\left[\mathrm{C}_{4} \mathrm{H}_{3}\right]^{+}$.

2-(2-Bromobenzyl)thio-5-(3,4-methylenedioxyphenyl)1,3,4-oxadiazole (6f): White amorphous solid; Yield: $82 \%$; m.p: $120{ }^{\circ} \mathrm{C}$; m.f.: $\mathrm{C}_{16} \mathrm{H}_{11} \mathrm{~N}_{2} \mathrm{O}_{3} \mathrm{SBr}$; Molecular mass: 391; IR $\left(\mathrm{KBr}, v_{\max }, \mathrm{cm}^{-1}\right.$ ): 3329 (aromatic C-H stretching), 1633 (Ar $\mathrm{C}=\mathrm{C}$ stretching $), 1580(\mathrm{C}=\mathrm{N}$ stretching $), 1108$ (C-O-C stretching); ${ }^{1} \mathrm{H} \mathrm{NMR}\left(\mathrm{CDCl}_{3}, 400 \mathrm{MHz}, \delta / \mathrm{ppm}\right): 7.62$ (dd, $J=7.6$, $\left.1.6 \mathrm{~Hz}, 1 \mathrm{H}, \mathrm{H}-3^{\prime \prime}\right), 7.58$ (d, $J=8.0 \mathrm{~Hz}, 1 \mathrm{H}, \mathrm{H}-6$ '), 7.50 (dd, $J=$ 8.0, 1.6 Hz, 1H, H-6"), 7.41 (d, $\left.J=1.6 \mathrm{~Hz}, 1 \mathrm{H}, \mathrm{H}-2^{\prime}\right), 7.18$ 7.12 (m, 2H, H-4", H-5"), 6.88 (d, J=8.0 Hz, 1H, H-5'), 6.03 (s, 2H, H-7'), 4.60 (s, 2H, H-7"); EIMS ( $m / z): 393[\mathrm{M}+2]^{\bullet+}$, $391[\mathrm{M}]^{\bullet+}, 221\left[\mathrm{C}_{9} \mathrm{H}_{5} \mathrm{~N}_{2} \mathrm{O}_{3} \mathrm{~S}\right]^{+}, 202\left[\mathrm{C}_{7} \mathrm{H}_{6} \mathrm{SBr}\right]^{+}, 189$ $\left[\mathrm{C}_{9} \mathrm{H}_{5} \mathrm{~N}_{2} \mathrm{O}_{3}\right]^{+}, 171\left[\mathrm{C}_{7} \mathrm{H}_{7} \mathrm{Br}\right]^{\bullet+}, 163\left[\mathrm{C}_{8} \mathrm{H}_{5} \mathrm{NO}_{3}\right]^{\bullet+}, 157$ $\left[\mathrm{C}_{6} \mathrm{H}_{5} \mathrm{Br}\right]^{\bullet+}, 147\left[\mathrm{C}_{8} \mathrm{H}_{5} \mathrm{NO}_{2}\right]^{\bullet+}, 121\left[\mathrm{C}_{7} \mathrm{H}_{5} \mathrm{O}_{2}\right]^{+}, 90\left[\mathrm{C}_{7} \mathrm{H}_{6}\right]^{+}, 77$ $\left[\mathrm{C}_{6} \mathrm{H}_{5}\right]^{+}, 65\left[\mathrm{C}_{5} \mathrm{H}_{5}\right]^{\bullet+}, 51\left[\mathrm{C}_{4} \mathrm{H}_{3}\right]^{+}$.

2-(3-Bromobenzyl)thio-5-(3,4-methylenedioxyphenyl)1,3,4-oxadiazole (6g): White amorphous solid; Yield: $85 \%$; m.p: $110{ }^{\circ} \mathrm{C}$; m.f.: $\mathrm{C}_{16} \mathrm{H}_{11} \mathrm{~N}_{2} \mathrm{O}_{3} \mathrm{SBr}$; Molecular mass: 391; IR $\left(\mathrm{KBr}, \mathrm{v}_{\max }, \mathrm{cm}^{-1}\right): 3290$ (aromatic $\mathrm{C}-\mathrm{H}$ stretching), 1628 (Ar $\mathrm{C}=\mathrm{C}$ stretching), 1587 (C=N stretching), 1107 (C-O-C stretching); ${ }^{1} \mathrm{H} \mathrm{NMR}\left(\mathrm{CDCl}_{3}, 300 \mathrm{MHz}, \delta / \mathrm{ppm}\right): 7.59$ (s, $1 \mathrm{H}, \mathrm{H}-$ 2"), 7.51 (dd, $J=8.1,1.5 \mathrm{~Hz}, 1 \mathrm{H}, \mathrm{H}-6$ '), 7.41 (brd.s, $1 \mathrm{H}, \mathrm{H}-$ 2'), 7.39-7.37 (m, 2H, H-4", H-6"), 7.18 (t, J=7.8 Hz, 1H, H5"), 6.89 (d, $\left.J=7.1 \mathrm{~Hz}, 1 \mathrm{H}, \mathrm{H}-5^{\prime}\right), 6.06$ (brd.s, 2H, H-7'), 4.43 (s, 2H, H-7"); EIMS (m/z): $393[\mathrm{M}+2]^{\bullet+}, 391[\mathrm{M}]^{\bullet+}, 221$
$\left[\mathrm{C}_{9} \mathrm{H}_{5} \mathrm{~N}_{2} \mathrm{O}_{3} \mathrm{~S}\right]^{+}, 202\left[\mathrm{C}_{7} \mathrm{H}_{6} \mathrm{SBr}\right]^{+}, 189\left[\mathrm{C}_{9} \mathrm{H}_{5} \mathrm{~N}_{2} \mathrm{O}_{3}\right]^{+}, 171$ $\left[\mathrm{C}_{7} \mathrm{H}_{7} \mathrm{Br}\right]^{\bullet+}, 163\left[\mathrm{C}_{8} \mathrm{H}_{5} \mathrm{NO}_{3}\right]^{\bullet+}, 157\left[\mathrm{C}_{6} \mathrm{H}_{5} \mathrm{Br}\right]^{{ }^{++}}, 147\left[\mathrm{C}_{8} \mathrm{H}_{5} \mathrm{NO}_{2}\right]^{\bullet+}$, $121\left[\mathrm{C}_{7} \mathrm{H}_{5} \mathrm{O}_{2}\right]^{+}, 90\left[\mathrm{C}_{7} \mathrm{H}_{6}\right]^{+}, 77\left[\mathrm{C}_{6} \mathrm{H}_{5}\right]^{+}, 65\left[\mathrm{C}_{5} \mathrm{H}_{5}\right]^{\bullet+}, 51\left[\mathrm{C}_{4} \mathrm{H}_{3}\right]^{+}$.

2-(4-Bromobenzyl)thio-5-(3,4-methylenedioxyphenyl)1,3,4-oxadiazole (6h): White amorphous solid; Yield: $93 \%$; m.p: $125^{\circ} \mathrm{C}$; m.f.: $\mathrm{C}_{16} \mathrm{H}_{11} \mathrm{~N}_{2} \mathrm{O}_{3} \mathrm{SBr}$; Molecular mass: 391; IR $\left(\mathrm{KBr}, v_{\max }, \mathrm{cm}^{-1}\right.$ ): 3450 (aromatic C-H stretching), 1627 (Ar $\mathrm{C}=\mathrm{C}$ stretching), $1578(\mathrm{C}=\mathrm{N}$ stretching), 1109 (C-O-C stretching); ${ }^{1} \mathrm{H} \mathrm{NMR}\left(\mathrm{CDCl}_{3}, 400 \mathrm{MHz}, \delta / \mathrm{ppm}\right): 7.50$ (dd, $J=8.0$, $\left.1.6 \mathrm{~Hz}, 1 \mathrm{H}, \mathrm{H}-6^{\prime}\right), 7.44$ (d, J = 8.4 Hz, 2H, H-3", H-5"), 7.40 (d, $\left.J=1.6 \mathrm{~Hz}, 1 \mathrm{H}, \mathrm{H}-2^{\prime}\right), 7.32$ (d, $J=8.4 \mathrm{~Hz}, 2 \mathrm{H}, \mathrm{H}-2 "$, H-6"), 6.88 (d, J=8.0 Hz, 1H, H-5'), 6.03 (s, 2H, H-7'), 4.42 (s, 2H, H-7"); $\operatorname{EIMS}(\mathrm{m} / \mathrm{z}): 393[\mathrm{M}+2]^{\bullet+}, 391[\mathrm{M}]^{\bullet+}, 221$ $\left[\mathrm{C}_{9} \mathrm{H}_{5} \mathrm{~N}_{2} \mathrm{O}_{3} \mathrm{~S}\right]^{+}, 202\left[\mathrm{C}_{7} \mathrm{H}_{6} \mathrm{SBr}\right]^{+}, 189\left[\mathrm{C}_{9} \mathrm{H}_{5} \mathrm{~N}_{2} \mathrm{O}_{3}\right]^{+}, 171$ $\left[\mathrm{C}_{7} \mathrm{H}_{7} \mathrm{Br}\right]^{\bullet+}, 163\left[\mathrm{C}_{8} \mathrm{H}_{5} \mathrm{NO}_{3}\right]^{\bullet+}, 157\left[\mathrm{C}_{6} \mathrm{H}_{5} \mathrm{Br}\right]^{{ }^{++}}, 147\left[\mathrm{C}_{8} \mathrm{H}_{5} \mathrm{NO}_{2}\right]^{\bullet+}$, $121\left[\mathrm{C}_{7} \mathrm{H}_{5} \mathrm{O}_{2}\right]^{+}, 90\left[\mathrm{C}_{7} \mathrm{H}_{6}\right]^{+}, 77\left[\mathrm{C}_{6} \mathrm{H}_{5}\right]^{+}, 65\left[\mathrm{C}_{5} \mathrm{H}_{5}\right]^{\bullet+}, 51\left[\mathrm{C}_{4} \mathrm{H}_{3}\right]^{+}$.

\section{Urease inhibition assay}

The urease enzyme inhibition assay is the modified form of Berthelot assay ${ }^{14}$.

\section{Statistical analysis}

The results were compiled in triplicate and presented as mean \pm sem. The statistical analysis was computed by ME 2010 .

\section{RESULTS AND DISCUSSION}

Eight 1,3,4-oxadiazole derivatives (6a-h), were proficiently synthesized in good yields as given in Scheme-I. The<smiles>O=C(O)c1ccc2c(c1)OCO2</smiles>

(1)<smiles>CCOC(=O)c1ccc2c(c1)O[C@H](C(N)=O)[C@H](C(=O)OCC)O2</smiles>

(2)

(3)

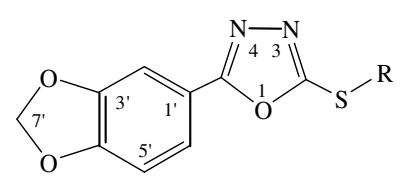

(6a-h)

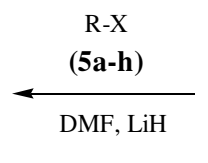

DiF, LiH \begin{tabular}{l|l}
$\mathrm{C}_{2} \mathrm{H}_{5} \mathrm{OH}$ & $\begin{array}{l}\mathrm{KOH}, \mathrm{CS}_{2} \\
\text { reflux }\end{array}$
\end{tabular}<smiles>Sc1nnc(-c2ccc3c(c2)OCO3)o1</smiles>

(4)

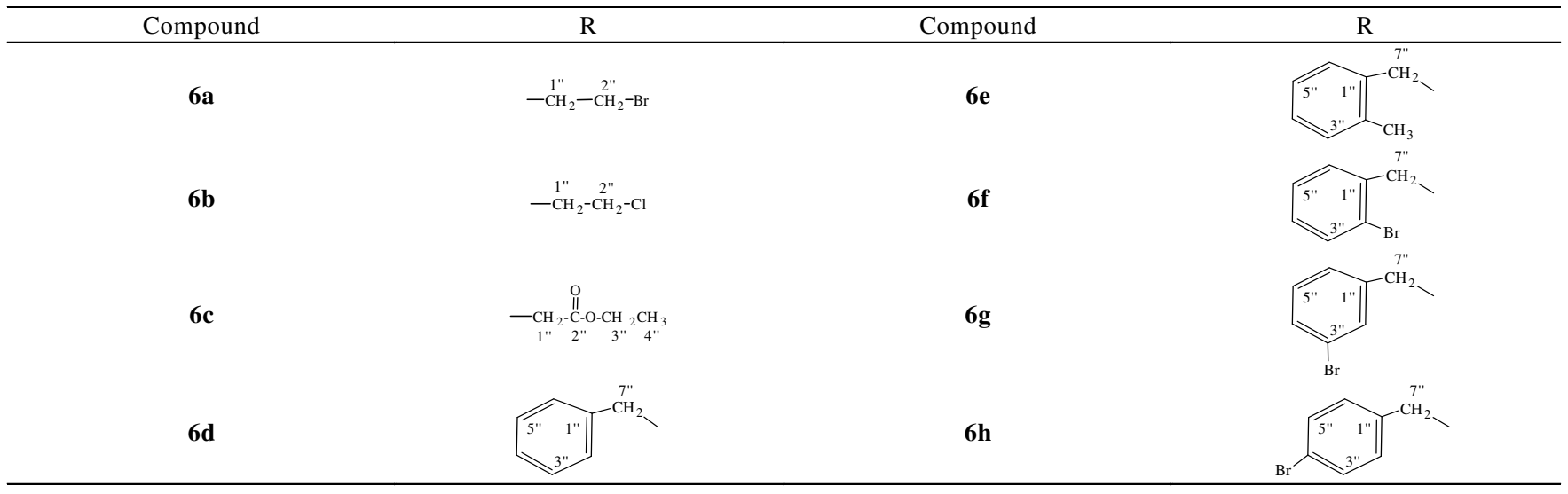


reaction specifications and the structural evaluating data are given earlier.

Our prompting accusative for taking this task was to synthesize new 1,3,4-oxadiazole bearing compounds but with potential against urease enzyme. The research work comprised of synthesis of 5-(3,4-methylenedioxyphenyl)-1,3,4oxadiazol-2-thiol (4) from 3,4-(methylenedioxy)benzoic acid (1) through three steps. Primary the ethyl ester, $\mathbf{2}$ of $\mathbf{1}$ was synthesized by refluxing with ethanol for $3 \mathrm{~h}$ using conc. $\mathrm{H}_{2} \mathrm{SO}_{4}$ as catalyst. Secondary, the corresponding hydrazide, $\mathbf{3}$ of $\mathbf{2}$ was yielded after a reflux of $6 \mathrm{~h}$ with $80 \% \mathrm{~N}_{2} \mathrm{H}_{4} \cdot \mathrm{H}_{2} \mathrm{O}$ in ethanol. Tertiary, $\mathbf{3}$ was intermolecularly cyclized to $\mathbf{4}$ via a reflux of 5 $\mathrm{h}$ in ethanol using $\mathrm{CS}_{2}$ as second reactant and $\mathrm{KOH}$ to basify the medium. The target 1,3,4-oxadiazole derivatives (6a-h) were yielded by stirring different alkyl/aralkyl halides (5a-h) with 4 in N,N-dimethyl formamide (DMF) in the presence of LiH for 4-6 h (Scheme-I). The structures of the yielded compounds were well confirmed by IR, ${ }^{1} \mathrm{H}$ NMR and EIMS spectral data.

Compound $\mathbf{6 a}$ was synthesized as creamy white amorphous solid in a good yield of $90 \%$ with m.p. $122{ }^{\circ} \mathrm{C}$. The molecular formula $\mathrm{C}_{11} \mathrm{H}_{9} \mathrm{~N}_{2} \mathrm{O}_{3} \mathrm{SBr}$ was set up by EI-MS with $[\mathrm{M}]^{\bullet+}$ peak at $329(\mathrm{~m} / \mathrm{z})$ and also by the protons resonating in ${ }^{1} \mathrm{H}$ NMR spectrum. The IR spectrum supported the 1,3,4oxadiazole structure by showing stretching bands at 1591-1576 $\mathrm{cm}^{-1}$ for $(\mathrm{C}=\mathrm{N})$ and at $1109-1106 \mathrm{~cm}^{-1}$ for $(\mathrm{C}-\mathrm{O}-\mathrm{C})$. The distinct peak in EI-MS at $221(\mathrm{~m} / \mathrm{z})$ was owing to 5-(3,4-methylenedioxyphenyl)-1,3,4-oxadiazol-2-thio cation. The ${ }^{1} \mathrm{H}$ NMR spectrum showed four signals, one doublet doublet, one doublet with small coupling, one doublet with large coupling and one singlet at $\delta 7.53\left(\mathrm{dd}, J=8.0,1.2 \mathrm{~Hz}, 1 \mathrm{H}, \mathrm{H}-6{ }^{\prime}\right), 7.43(\mathrm{~d}, J=$ $\left.1.2 \mathrm{~Hz}, 1 \mathrm{H}, \mathrm{H}-2^{\prime}\right), 6.89$ (d, $J=8.0 \mathrm{~Hz}, 1 \mathrm{H}, \mathrm{H}-5$ ') and 6.04 (s, $\left.2 \mathrm{H}, \mathrm{H}-7^{\prime}\right)$ were attributed to methylenedioxyphenyl ring; and two triplets at $\delta 3.76(\mathrm{t}, J=7.2 \mathrm{~Hz}, 2 \mathrm{H}, \mathrm{H}-2 ")$ and $3.68(\mathrm{t}, J=$ $7.2 \mathrm{~Hz}, 2 \mathrm{H}, \mathrm{H}-1 ")$ owed to bromoethyl group in the molecule. All this demonstrated the structure of $\mathbf{6 a}$ as 2-(2-bromoethyl)thio-5-(3,4-methylenedioxyphenyl)-1,3,4-oxadiazole. The structures of other molecules were also corroborated by spectral data given in experimental section. The mass fragmentation pattern of 2-(2-bromobenzyl)thio-5-(3,4-methylenedioxyphenyl)-1,3,4-oxadiazole (6f) is sketched in Fig. 1.

Urease enzyme inhibition activity (in vitro): in vitro urease inhibition activity results for all the synthesized molecules are tabulated as \%age inhibition and $\mathrm{IC}_{50}$ values (Table-1). All the compounds were also screened against urease enzyme and showed varying degree of enzyme inhibition from moderate to excellent as evident from Table-1. The synthesized compounds $\mathbf{6 a}, \mathbf{6 b}, \mathbf{6 e}, \mathbf{6 g}$ and $\mathbf{6 h}$ showed relatively better inhibition potential. The molecule $\mathbf{6 g}$ exhibited almost the same inhibition activity as that of the reference standard. It showed the $\mathrm{IC}_{50}$ value of $30.10 \pm 0.65 \mu \mathrm{M}$ with respect to thiourea, the reference standard with $\mathrm{IC}_{50}$ value of $21.28 \pm 0.11 \mu \mathrm{M}$. The promising activity of $\mathbf{6} \mathbf{g}$ was probably because of the presence of meta-substituted bromoaralkyl group (the halogenated group). The molecule $6 \mathbf{e}$ displayed two times less activity relative to reference as by $\mathrm{IC}_{50}$ values. Two synthesized compounds, $6 \mathbf{a}$ and $\mathbf{6 h}$ depicted same inhibitory potential; three times less than that of the reference standard. The structural

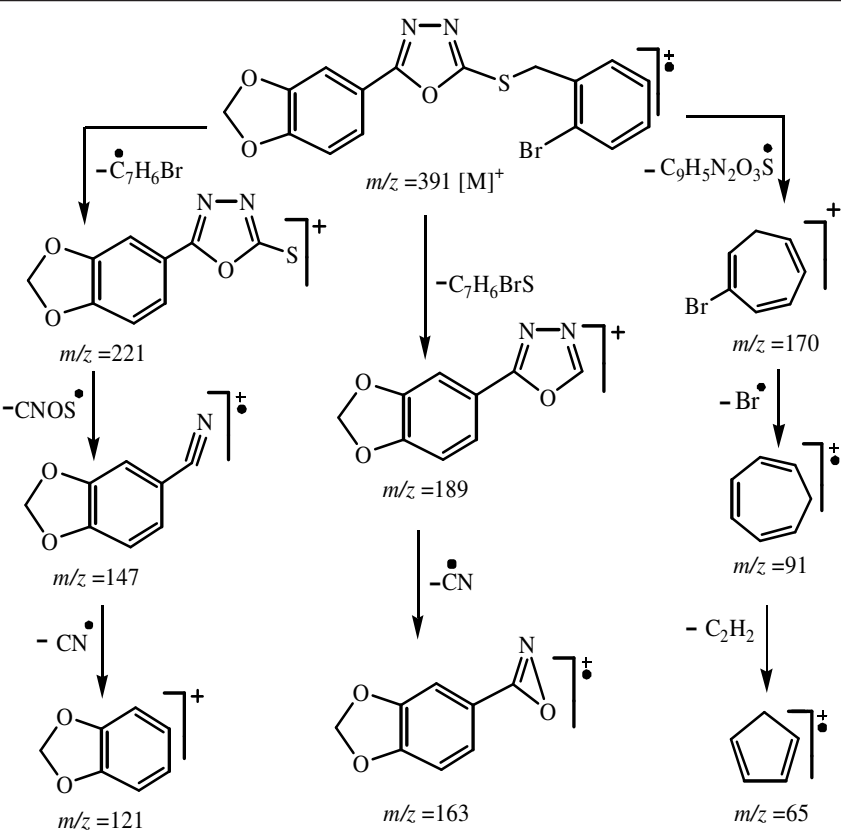

Fig. 1. Mass fragmentation pattern of 2-(2-bromobenzyl)thio-5-(3,4methylenedioxyphenyl)-1,3,4-oxadiazole (6f)

\begin{tabular}{|c|c|c|}
\hline \multicolumn{3}{|c|}{$\begin{array}{l}\text { TABLE-1 } \\
\text { UREASE ENZYME INHIBITION STUDY } \\
\text { OF THE SYNTHESIZED COMPOUNDS }\end{array}$} \\
\hline \multirow{2}{*}{ Compound } & \multicolumn{2}{|c|}{ Urease enzyme } \\
\hline & \%age Inhibition & $\mathrm{IC}_{50}(\mu \mathrm{M})$ \\
\hline 6a & $70.90 \pm 0.67$ & $68.20 \pm 0.91$ \\
\hline $\mathbf{6 b}$ & $97.0 \pm 0.79$ & $313.49 \pm 0.56$ \\
\hline $6 c$ & $6.50 \pm 0.19$ & - \\
\hline 6d & $27.90 \pm 0.46$ & - \\
\hline $6 e$ & $61.36 \pm 0.97$ & $46.20 \pm 0.85$ \\
\hline $6 f$ & $12.40 \pm 0.51$ & - \\
\hline $6 \mathrm{~g}$ & $96.10 \pm 0.34$ & $30.10 \pm 0.65$ \\
\hline $6 \mathrm{~h}$ & $95.90 \pm 0.29$ & $68.50 \pm 1.40$ \\
\hline Thiourea & $98.61 \pm 0.77$ & $21.28 \pm 0.11$ \\
\hline \multicolumn{3}{|c|}{$\begin{array}{l}\text { Note: } \mathrm{IC}_{50} \text { values (concentration at which there is } 50 \% \text { enzyme } \\
\text { inhibition) of compounds were calculated using EZ-Fit Enzyme } \\
\text { kinetics software (Perella Scientific Inc. Amherst, USA) }\end{array}$} \\
\hline
\end{tabular}

overview of these potent compounds rendered them as halogen bearing molecules. The order of the enzyme inhibition activity of these molecules was found to be as:

$$
6 \mathrm{~g}>6 \mathrm{e}>6 \mathrm{a}>6 \mathrm{~h}>6 \mathrm{~b}
$$

\section{Conclusion}

The synthesized products were developed in better yields with ground support of spectroscopic data. The basic aim of this synthetic work was to search out potent inhibitors of urease enzyme and the attempt remained fruitful to much extent. Out of eight synthesized molecules, five were found to be potential inhibitor of urease enzyme with relatively very low $\mathrm{IC}_{50}$ values. These molecules because of anti-urease potential, may assist in drug designing to the pharmaceutical industries.

\section{ACKNOWLEDGEMENTS}

Special thanks are paid to the Higher Education Commission of Pakistan for the financial aid. 


\section{REFERENCES}

1. B. Roy, A. Chakraborty, S.K. Ghosh and A. Basak, Bioorg. Med. Chem. Lett., 19, 7007 (2009).

2. R.R. Somani and P.Y. Shirodkar, Der Pharm. Chem., 1, 130 (2009).

3. N. Jaiswal, A.K. Singh, D. Singh and T. Ahmad, Int. Res. J. Pharm., 3, 83 (2012).

4. Aziz-ur-Rehman A. Fatima, N. Abbas, M.A. Abbasi, K.M. Khan, M Ashraf, I. Ahmad and S.A. Ejaz, Pak. J. Pharm. Sci., 26, 345 (2013).

5. Aziz-ur-Rehman, A. Fatima, M.A. Abbasi, S. Rasool, A. Malik, M. Ashraf, I. Ahmad and S.A. Ejaz, J. Saudi Chem. Soc., doi:10.1016/j.jscs.2013. 02.006.

6. H. Khalid, Aziz-ur-Rehman, M.A. Abbasi, A. Malik, S. Rasool, K. Nafeesa, I. Ahmad and S. Afzal, J. Saudi Chem. Soc., doi:10.1016/j.jscs.2013. 05.001.

7. L. Ingrassia, F. Lefranc, V. Mathieu, F. Darro and R. Kiss, Translational Oncol., 1, 1 (2008).
8. B. Bar-Oz, T. Einarson, A. Einarson, R. Boskovic, L. O'Brien, H. Malm, A. Berard and G. Koren, Clin. Ther., 29, 918 (2007).

9. H.L.T. Mobley, M.D. Island and R.P. Hausinger, Microbiol. Rev., 59, 451 (1995).

10. M.A. Lodhi, M.A. Abbasi, M.I. Choudhary and V.U. Ahmad, Nat. Prod. Res., 21, 721 (2007).

11. M.A. Lodhi, J. Hussain, M.A. Abbasi, A.R. Jassbi, M.I. Choudhary and V.U. Ahmad, J. Enzyme Inhib. Med. Chem., 21, 531 (2006).

12. Aziz-ur-Rehman S.Z. Siddiqui, N. Abbas, M.A. Abbasi, K.M. Khan, M. Shahid, Y. Mahmood, N.M. Akhtar and N.H. Lajis, Int. J. Pharm. Pharm. Sci., 4, 676 (2012).

13. Aziz-ur-Rehman K. Nafeesa, M.A. Abbasi, H. Khalid, K.M. Khan, M. Ashraf, I. Ahmad and S.A. Ejaz, Asian J. Pharm. Heath Sci., 2, 370 (2012).

14. H.L. Mobley, M.J. Cortesia, L.E. Rosenthal and B.D. Jones, J. Clin. Microbiol., 26, 831 (1988). 